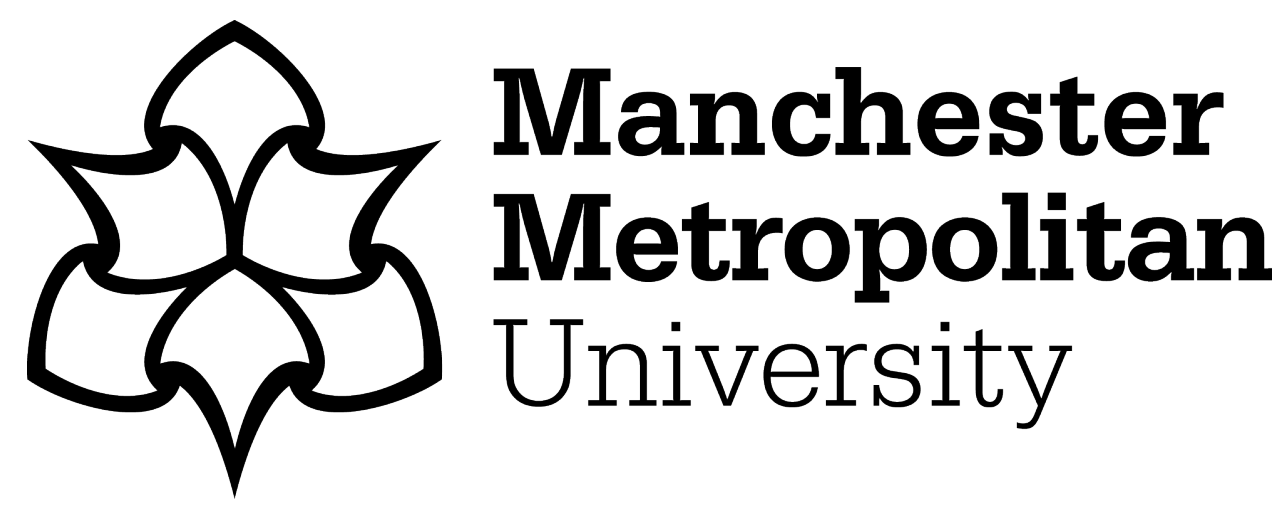

Orlando, G, Pinedo-Villanueva, R, Reeves, ND, Javaid, MK and Ireland, A ORCID logoORCID: https://orcid.org/0000-0003-1094-9183 (2021) Physical function in UK adults with osteogenesis imperfecta: a cross-sectional analysis of the RUDY study. Osteoporosis International, 32 (1). pp. 157-164. ISSN 0937-941X

Downloaded from: https://e-space.mmu.ac.uk/626834/

Version: Accepted Version

Publisher: Springer (part of Springer Nature)

DOI: https://doi.org/10.1007/s00198-020-05537-3

Please cite the published version 


\section{Physical function in UK adults with osteogenesis imperfecta: a cross- sectional analysis of the RUDY study}

Giorgio Orlando ${ }^{1}$, Rafael Pinedo-Villanueva ${ }^{2}$, Neil D Reeves ${ }^{1}$, Muhammad Kassim Javaid ${ }^{2}$, and Alex Ireland ${ }^{1}$

${ }^{1}$ Research Centre for Musculoskeletal Science \& Sports Medicine, Department of Life Sciences, Manchester Metropolitan University, Manchester, United Kingdom; ${ }^{2}$ Nuffield Department of Orthopaedics, Rheumatology and Musculoskeletal Sciences, Oxford NIHR Musculoskeletal Biomedical Research Unit, Rheumatology and Musculoskeletal Sciences, University of Oxford, Oxford, UK

\section{Corresponding Author:}

Giorgio Orlando, $\mathrm{PhD}$,

Research Centre for Musculoskeletal Science \& Sports Medicine,

Department of Life Sciences,

Manchester Metropolitan University,

John Dalton Building, Chester Street, Manchester, M1 5GD.

E-mail: G.Orlando@mmu.ac.uk 


\begin{abstract}
Summary We describe physical function in adults with osteogenesis imperfecta (OI) and explored clinical and non-clinical factors related to its impairment. Our data showed that physical dysfunction is a common feature of adults with OI, varying by OI severity, and mediated by the presence and quality of pain and fatigue symptoms.
\end{abstract}

Introduction There is a paucity of data describing physical function in adults with Osteogenesis imperfecta (OI). We investigated the effects of OI and its severity on physical function, and explored the relationship between physical function and number of fractures and symptomatology.

Methods Adults with OI of different types were recruited from the RUDY study, an ongoing UKbased prospective cohort study. Participants completed demographic and clinical questions, and questionnaires. These assessed physical function (SF-36), mobility (EQ-5D-5L and NEADL), fatigue (FACIT-F) and pain (SF-MQ-2). Scores were compared using parametric or non-parametric statistical analyses, whereas correlations between outcomes were examined using univariate and multivariate regression analysis.

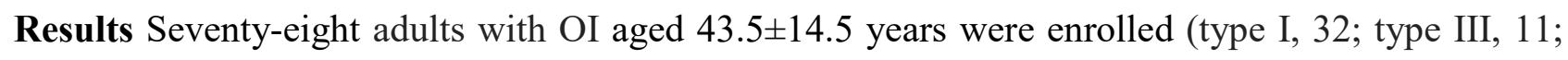
type IV, 10; unknown type, 26). Physical function (PCS, SF-36) was significantly lower in all participants than normative values $(p<0.001)$ and in type III than type I $(p=0.008)$. Mobility was significantly different across the types (EQ-5D-EL, $p=0.007$; NEADL, $p<0.001$ ), with type III having more severe problems, followed by types IV, unknown and I. Physical function was associated with OI type $(r=0.26 ; p=0.021)$, presence and quality of pain $(r=-0.57 ; p<0.0001)$ and fatigue $(r=-0.51$; $\mathrm{p}<0.0001)$. Multivariate analysis revealed that physical function correlated independently with age, OI type, fatigue and non-neuropathic pain. 
Conclusions Individuals with OI display a marked deterioration in physical function during adulthood. This impairment varies in severity according to the OI phenotype and is associated with the presence of non-neuropathic pain and fatigue. 


\section{Introduction}

Osteogenesis imperfecta (OI) is a genetic skeletal disorder, characterised by qualitative and quantitative abnormalities of type I collagen[1]. In approximately $80 \%$ of individuals with OI, a mutation in one of the COL1A1 or COL1A2 genes is believed to be responsible for the disorder ${ }^{1}$. Although the incidence of OI varies globally, it has been estimated to occur in 1 in 10,000 to 1 in 20,000 births. In the United Kingdom, it has been reported that approximately 3,400 individuals have this condition[2].

The main clinical feature of OI is decreased bone mineral density and bone fragility, leading to a high incidence of fractures, particularly in childhood[1]. The chronic sequelae of fractures also causes a higher incidence rate of osteoarthritis and skeletal deformities, predisposing the patient to chronic pain[3]. Such extreme debilitating symptoms, in concert with fatigue, may be linked to physiological stress and physical inactivity[4]. As a consequence, the clinical condition of people with $\mathrm{OI}$ is related to both disease and inactivity.

Among the array of OI clinical features, musculoskeletal manifestations are the main determinants of physical deterioration and quality of life (QoL). There is also a growing body of evidence suggesting that OI patients display lower muscle strength and power and greater fatigability[5]. These characteristics are associated with reduced muscle mass and quality (i.e. strength per unit of muscle mass)[5].

While the effect of OI on physical function has been largely explored in children and adolescents, limited investigations have been conducted in adults[6-8]. Among this cohort, the impact of OI on physical function remains poorly described, with no investigations conducted on the UK adult population. Additionally, there is no uniformity of evidence about the relationship between the OI type and severity of physical function impairment.

Our aim was to describe in adults with OI, the differences in physical function by OI type and the relationship between physical function and history of fractures, pain and fatigue symptoms. We 
hypothesise that physical function is impaired in people with OI during adulthood according to its severity, and that these deficits are mediated by the number of fractures and specific symptoms.

\section{Methods}

\section{Participants}

This cross-sectional analysis used data from the Rare and Undiagnosed Diseases Study (RUDY study), an ongoing UK-based multi-centre prospective cohort study, aimed at improving the understanding of rare diseases affecting musculoskeletal systems[9]. Data were collected using a web-based registry and patient-driven research platform. The RUDY web-platform has been previously described[9]. Briefly, patients gave online dynamic consented using the RUDY website (www.rudystudy.com) and were then given access to their own password-protected website that included validated questionnaires and forms for the participant to record OI type and medical events (i.e. number of fractures). Participants were invited every six months to complete questionnaires through email messages sent from the RUDY platform.

For this analysis, inclusion criteria were a self-reported diagnosis of OI from participants aged 18 or over and completed baseline questionnaires.

\section{Patient-reported outcome measures}

Participants were asked to respond to general questions regarding demographic information, OI type, and the number of fractures during their lifetime. Participants self-reported their OI type if known, but were also able to select type ‘unknown'. Self-reported questionnaire-based methods are accurate for the assessment of fractures, particularly for those occurring at the clinically-relevant hip and distal radius sites [10]. In addition, participants were asked to complete five questionnaires: general health status (The Short-Form 36 health survey (SF-36) and the EuroQol five-dimensional questionnaire (EQ-5D-5L), fatigue (The functional assessment of chronic illness therapy fatigue 
(FACIT-F), pain (Short-Form McGill Pain Questionnaire 2 (SF-MPQ-2) and functional performance during daily living activities (Nottingham Extended Activities of Daily Living (NEADL).

The SF-36 version 1.0 questionnaire is composed of 36 items measuring four domains of physical function, four domains of mental status, and is completed with reference to the previous four weeks[11]. Scoring ranges from 0 which corresponds to the worst possible status, to 100 which is the best possible status. Physical and mental domains are combined to calculate the physical component score (PCS) and mental component score (MCS)[11]. Scores were generated by multiplying each SF36 scale z-score, by its respective physical factor or mental component scoring coefficient, and summing the eight products. Reference values and scoring coefficients were based on general UK population data provided by Jenkison et al $[12,13]$. The component scores were then transformed so that each had a mean of 50, and a standard deviation (SD) of 10. The SF-36 version 1.0 provides six response levels to question 21 (bodily pain), whereas only five options, with the exclusion of "very mild", were shown to participants in our web-form. We therefore randomly allocated the 22 participants answering "mild" into two groups, corresponding to response levels of "very mild' and "mild", respectively, and thereafter calculating scores according to the traditional procedure.

The EQ-5D-5L instrument describes health-related QoL in terms of five dimensions: mobility, self-care, usual activities, pain/discomfort and anxiety/depression[14]. Each dimension has five levels of functioning: (1) no problems; (2) slights problems; (3) moderate problems, (4) severe problems and (5) unable/extreme problems. For a better understanding, response levels for each dimension are presented in the manuscript divided into three categories: 'no and slight problems` (levels 1 and 2), ‘moderate' (level 3) and, 'severe and extreme problems` (levels 4 and 5). Reference values were based on UK population data[15].

The SF-MPQ-2 tool characterises pain quality and intensity[16]. It consists of 22 items grouped into four dimensions (continuous, intermittent, neuropathic and affective pains). Each item is rated based on a $0-10$ scale, where 0 is 'no pain' and 10 is ‘worst pain'. Continuous and intermittent scores were combined to assess non-neuropathic pain. Three scores (neuropathic, non-neuropathic 
and affective) were calculated from the mean of the items included in each dimension and a total score by the mean of all items.

FACIT-F assesses the fatigability of different domains including physical, social/family, functional, wellbeing and additional concerns[17]. It uses a five-point Likert scale $(0=$ 'not at all' to 4 = 'very much'). Item scores were transformed based on FACIT guidelines and then combined to obtain a total summative score, with higher scores reflecting a lower fatigue level. The value set for the USA was used as normative data[18].

NEADL quantifies (22 items of) functional independence in four areas: mobility, kitchen, domestic and leisure[19]. The scores of each item were combined to give four subscales scores, while the sum of each item gave a total score. Higher scores represent better function and therefore greater independence.

\section{Statistical Analysis}

Data were expressed as the mean $\pm \mathrm{SD}$ for parametric variables, median and interquartile range (IQR) for nonparametric data, and percentages for categorical variables. All parameters were tested for normal distribution by visual inspection and Kolmogorov-Smirnov test. Differences among the OI groups in terms of age, number of fractures and scores from SF-36, NEADL, FACIT-F and SFMPQ-2 questionnaires were assessed using analysis of variance (ANOVA) for parametric variables, or the corresponding Kruskal-Wallis tests for nonparametric continuous variables, and the $\chi^{2}$ test for categorical variables. When indicated, the Tukey's (parametric) or Dunn's (nonparametric) post hoc tests for multiple comparisons were applied.

Analysis of covariance (ANCOVA) was used to test differences in number of fractures among the OI groups after adjusting for age. Comparisons between SF-36, FACIT-F and EQ-5D-5L scores and normative data were performed using one-sample $t$ tests. Fisher's exact tests were performed to assess differences among OI types on the proportion of participant responses for each category of the EQ-5D-5L questionnaire. 
Univariate correlation analyses between PCS and age, number of fractures, presence and quality of pain (non-neuropathic, neuropathic and affective pain), and fatigue were performed using Pearson's or Spearman's rho test. Multivariate linear regression analyses with stepwise backward selection were applied to assess independent correlates of PCS. Covariates were age, OI type, sex, number of fractures, fatigue and quality of pain. Regression imputation following the EM algorithm was used to replace 24 missing values of the variable 'number of fractures'. This data was then included in the multiple regression model.

\section{Results}

Baseline characteristics

Of the 150 adults with OI (47 males, 103 females, aged $42.8 \pm 14.5$ years) who were recruited into the RUDY study, 78 individuals (20 males and 58 females), with a mean age of $43.5 \pm 14.5$ years, fully completed all questionnaires (response rate of 52\%). Adults with OI who did not complete the questionnaires included 27 males and 45 females, with a mean age of $42.1 \pm 14.5$ years and different types of OI (29 type I, 11 type III, 4 type IV, 3 type V and 25 unknown type). No differences were found in age, sex, or OI types distribution among people who did and did not complete the questionnaires.

The clinical characteristics of study participants are shown in Table 1. Thirty-two (41\%) individuals reported a diagnosis of OI type I, 10 (12.8\%) of type III, and 10 (12.8\%) of type IV and $26(33.3 \%)$ an unknown type. Participants with OI type III were younger $(34.6 \pm 11.3$ years $)$ than those with type I $(45.7 \pm 14$ years; $p=0.025)$ and unknown type $(45.9 \pm 14.6$ years; $p=0.039)$, whereas no difference was observed with participants with type IV ( $40.7 \pm 18.8$ years; $p=0.392)$.

Fifty-four adults with OI (response rate of 69.2\%) provided information on the number of fractures during their lifetime (Table 1). From these participants, an average number of $26(\mathrm{SD} \pm 20.4)$ fractures per participant were reported in individuals with type III; 13 (SD \pm 8.7 ) in those with type I; 11.2 (SD 
$\pm 12.4)$ in those with type IV; and 16.5 (SD \pm 23.5$)$ in those with unknown type. Although there were large differences among the OI groups these were not statistically significant.

\section{Health status and functional independence}

Table 2 shows the physical domains and PCS scores from the SF-36 questionnaire. The mean values from physical domains and PCS scores were significantly lower in all participants, when compared to normative UK values $(\mathrm{p}<0.05)$. Among the OI types, participants with OI type I had significantly higher mean physical function $(P F)$ values than those with III $(p<0.001)$, IV $(p=0.038)$ and unknown OI types ( $\mathrm{p}=0.004)$, whereas only PCS was higher than those with type III $(\mathrm{p}=0.008)$. There were no statistically significant differences in the other outcome measures. Table 3 shows the total and subscale ADL scores according to the OI types. Participants with types III and IV had severe problems or were unable to perform most of the mobility and domestic tasks. Individuals with type I reported no problems or only mild problems performing most activities, whereas those with type unknown only had severe difficulties during domestic activities. Comparisons among the groups only showed significantly lower scores for mobility and domestic tasks among individuals with type III compared with those with type I $(\mathrm{p}<0.001)$.

Figure 1 shows the response level frequencies from the EQ-5D-5L questionnaire. Adults with OI reported more problems in mobility and usual activities and had more frequent pain symptoms than the general UK population $(\mathrm{p}<0.05)$. Self-care problems were more common in participants with types III $(p<0.001)$, IV $(p=0.023)$ and unknown $(p=0.009)$, whereas Anx/Depres was more frequent in types IV $(p<0.001)$ and unknown $(p=0.005)$ than the general UK pupulation. Among OI types, severe/extreme mobility problems were most commonly reported by adults with OI type III, followed by those with type IV, unknown type, and type I. Moderate mobility problems were reported by approximately $20 \%$ of participants with types III, IV and unknown, whereas a lower percentage of participants with type I reported such problems. Self-care was reported as not being/a slight problem for most participants with OI types I, IV, and unknown. This was in contrast to more than half of 
adults with type III who identified self-care as a problem. The majority of participants with type I indicated no/slight problems when performing usual activities. Severe/extreme problems were more frequent in individuals with type III, while moderate problems were more commonly reported in those participants with an unknown type. A similar percentage of participants in all groups reported severe/extreme pain, whereas moderate levels were most commonly found in type III patients, followed by types IV, unknown and I. Anxiety/depression was the least problematic issue for participants, with only a small proportion of respondents of all groups detailing severe or extreme anxiety/depression problems. The Fisher`s exact test detected significant differences among OI types in responses for the mobility $(\mathrm{p}=0.007)$ and self-care $(\mathrm{p}=0.049)$ dimensions and no differences for pain/discomfort $(\mathrm{p}=0.235)$ and anxiety/depression $(\mathrm{p}=0.384)$.

\section{Pain and fatigue}

Mild levels of neuropathic, non-neuropathic and affective pains were reported by all participants (Table 1). Non-neuropathic $(\mathrm{p}=0.005)$ and affective $(\mathrm{p}=0.002)$ pain scores were significantly higher than neuropathic pain. No significant differences were detected in subscales and total pain scores among OI types.

The FACIT-F score (Table 1) was significantly lower in all participants compared with the USA normative values $(\mathrm{p}<0.001)$. Although there were large differences across the OI types these were not statistically significant.

\section{Correlations}

Univariate analysis (Table 4) revealed that PCS was correlated with FACIT-F score (fatigue), and inversely with OI types, and non-neuropathic, neuropathic, affective and total pain scores. No correlation was found between PCS and age, sex and number of fractures. After adjusting for covariates, multivariate analysis (Table 4) showed that PCS was independently correlated with OI type and FACIT-F score and inversely with non-neuropathic pain score. 


\section{Discussion}

To the best of our knowledge, this is the first study to describe physical function in a cohort of UK adults with OI and across different OI types, and more specifically the first to assess associations between clinical problems such as fractures, pain and fatigue and deficits in physical function. The most salient results show that the impairment of physical function is a typical feature of adults with OI, which differs between OI types. Independent of this type-specific variation, specific symptoms such as non-neuropathic pain and fatigue are associated with lower physical function. These findings support the concept that phenotype and symptomatology contribute independently to the loss of functional independence in OI.

Although insufficient study power may have limited the capacity to detect differences in some parameters across the OI types, our data suggest that impaired physical function, and in particular of mobility affects individuals with OI type III, followed by those with type IV and then type I. These findings are confirmed by the association reported by multivariate analysis which revealed that OI type is an independent correlate of the impairment of physical function in OI. These results are in line with several studies exploring the effects of OI on surrogate measures of physical function in populations of different age. There is evidence showing that OI type is the most important determinant of the physical performance in childhood, adolescence and young adults [20,21]. A recent study conducted in a Danish adult population showed that physical health varies according to the type of OI, with marked impairment in people with type III than other types [4]. Taken together, findings from the present and previous studies support the hypothesis that individuals with OI are characterised by an impairment of physical function which persists across the lifespan, and the severity of functional impairment is dependent on OI type. The marked differences in physical function across the OI types likely result from the wide array of clinical manifestations (i.e. growth deficiency, degree of skeletal dysplasia and frequency of fractures) which vary significantly in both presence and severity according to the phenotype $[1,2]$. 
Since we reported large differences between physical outcomes and general UK population, we also suggest that physical function could be more markedly impaired than previously assumed. In particular, adults with type I reported an approximatively $25 \%$ lower physical function and mobility compared with the UK general population. These findings suggest that not only "moderate and severe" forms but also "mild" of OI determine marked impairment in physical performance during adulthood. However, more accurate measures need to be performed to quantify the amount of difference in physical function between healthy and OI adults. Although there is evidence showing a comparable level of function between types I and IV, our data promote the concept that individuals with type IV had values of physical function between types III and I [7, 21]. Since age and assessed measures were similar across the studies, it is possible that differences in lifestyle and health care between different study populations (USA vs UK) might have mediated this discrepancy.

In addition to OI type, our work is the first characterising the role played by fractures and specific symptoms such as fatigue and pain. Multivariate analysis, after accounting for covariates and missing data, showed the absence of association between the number of fractures and physical performance, thus suggesting that other factors may contribute to the high level of physical impairment in OI. Although this finding is difficult to reconcile with the extreme debilitating sequelae induced by fractures including, fear of fractures, pain and deformities, it is possible to hypothesise that the number of fractures could play a lesser role in the development of physical alterations than that reported in children. This could be related to the lower incidence of fractures[1] during adulthood than childhood, which might result in a longer recovery time and consequently in a partial restoration of the physical function and alleviating of the psychological distress.

Although it is well recognised that pain is an extremely debilitating symptom of OI responsible for the impairment of physical performance, we were the first to investigate the role played by the type of pain through the characterisation of the effect of non-neuropathic and neuropathic pain. Our data show that, although both types of pain are strongly associated with physical function (univariate correlation), only non-neuropathic pain was independently correlated (multivariate analysis) with 
physical function. Thus, we suggest that the quality of pain has a determinant role in the development of physical disability in adults with OI. Since only mild levels of pain were reported by the participants, we also suggest that chronic pain, independent of severity, has the capacity to induce severe functional deficits in OI. Our data shows that adults with OI have a higher level of fatigue than healthy individuals, and the severity of this symptom is not related to OI phenotype. These findings are in line with a recent study conducted in a Netherlands adult cohort with OI documenting high levels of fatigue performing several daily living activities[22]. This evidence supports the notion that fatigue is a common symptom in OI which plays a determinant role in the loss of physical function, highlighting the necessity to prevent or treat the symptom to counteract physical dysfunction in OI.

One potential impact of these findings is to highlight the importance of incorporating exercise into the care of adults with OI. Exercise training is the only strategy that has the potential to elicit multiple beneficial effects at the metabolic, cardiovascular, muscular and skeletal levels[23]. There is also evidence showing that exercise can alleviate pain symptoms and improve physical and physiological aspects related to the increased fatigability[24]; altogether, these adaptations showed the potential role of exercise on improving physical and mental health and therefore QoL for people with OI. Although the effects of exercise have been well documented in several clinical populations, currently the evidence available in OI has been gathered only in children and adolescents[25], thus there remains lack of information on the safety and effectiveness of exercise training for counteracting physical dysfunction and symptomatology in adults with OI and training for specialist physiotherapists and other allied health professionals.

This study presents several strengths and limitations. Its main strengths are the inclusion of participants with different OI types and the characterisation of physical function and symptomatology through the use of different validated tools. Limitations include the accuracy of self-reported measures, the partial specificity of the questionnaires used to explore physical function and symptoms in OI, the lack of a healthy control group and the cross-sectional design of the study, which did not allow assessment of the causal relationship between OI type, symptomatology and physical 
dysfunction. There are currently no OI-specific questionnaires for physical function, mobility and pain, and future development of OI-specific instruments is warranted. A minority of patients selfreported fractures which, combined with the substantial within-group variation in fracture incidence, may have limited our ability to detect group differences or associations with physical function. Individuals with OI self-reported their OI type in line with previous questionnaire-based studies, and we included an option for 'unknown' type for those who were uncertain. Despite this, there remains the possibility that individuals misreported their OI type which would decrease our power to detectgroup differences. In addition, a conservative method of statistical analyses was applied which may have limited our power to detect moderate differences in outcomes between individuals with different OI types. There was a geographical discrepancy between our SF-36 version and the UK version $[12,13]$ used to calculate scores for mental components, but as our study aims focused on physical rather than mental health this would not influence the main findings. Additionally, insufficient study power has limited the capacity to detect differences in some parameters. However, it is important to note that the small numbers of participants in types III and IV reflect not only the rarity of OI, but also the lower incidence of these types, which makes their enrolment even more complicated. As RUDY participants are self-selected, we cannot guarantee that it is representative of the OI population in the UK; however, it is the largest cohort of its type in the country and provides the best evidence possible to answer the questions we set out to examine. Finally, the response rate in our study was in line with previously published questionnaire-based studies, and assessment of participant characteristics of those individuals who did and did not complete the questionnaires were similar. Despite this, the limited numbers of responders and possible self-selection bias may also affect the generalisability of our findings.

In summary, this study reports cross-sectional data from a large cohort of the UK adults with OI, showing that the severity of physical impairment varies according to OI type. The presence and quality of specific symptoms such as non-neuropathic pain and fatigue are factors which contribute significantly to the development of such deficits. Specific countermeasures should be included in the 
standard care of adults with OI to prevent and counteract the loss of physical function and to alleviate pain and fatigability symptoms.

\section{Declarations}

Conflict of interest: G.O. was funded by a research grant from the Brittle Bone Society, R.P.V. receives consulting fees from Mereo Biopharma, all outside of the scope of this project. M.K.J. is a component of advisory board fee of Mereo Biopharma.

Ethical approval and consent to participate: This study was approved by the South Central Research Ethics committee UK after review (LREC 14/SC/0126) and participants gave informed consent.

Authors' contributions: Conception and design: G.O., R.PV., M.K.J. and A.I. Data acquisition or analysis and interpretation: G.O., M.K.J., A.I. Drafting the manuscript: G.O. Revising the manuscript critically for important intellectual content: all authors. Approved the final version: all authors. G. O. is responsible for the integrity of the work as a whole. 


\section{Figure Legend}

Fig. 1 Percentage frequency distributions of EQ-5D-5L dimensions in adults with OI and the general UK population 


\section{References}

1. Marini JC (2018) Osteogenesis imperfecta. Prim Metab Bone Dis Disord Miner Metab 3:871877. https://doi.org/10.1002/9781119266594.ch113

2. Martin E, Shapiro JR (2007) Osteogenesis imperfecta:epidemiology and pathophysiology. Curr Osteoporos Rep 5:91-7. https://doi.org/10.1136/jcp.49.8.627

3. Roberts TT, Cepela DJ, Uhl RL, Lozman J (2016) Orthopaedic Considerations for the Adult with Osteogenesis Imperfecta. J Am Acad Orthop Surg 24:298-308. https://doi.org/10.5435/JAAOS-D-15-00275

4. Tosi LL, Oetgen ME, Floor MK, et al (2015) Initial report of the osteogenesis imperfecta adult natural history initiative. Orphanet J Rare Dis 10:1-12. https://doi.org/10.1186/s13023-0150362-2

5. Veilleux LN, Trejo P, Rauch F (2017) Muscle abnormalities in osteogenesis imperfecta. J Musculoskelet Neuronal Interact 17:1-7

6. Widmann RF, Bitan FD, Laplaza FJ, et al (1999) Spinal deformity, pulmonary compromise, and quality of life in osteogenesis imperfecta. Spine (Phila Pa 1976) 24:1673-1678. https://doi.org/10.1097/00007632-199908150-00008

7. Hald JD, Folkestad L, Harsløf T, et al (2017) Health-Related Quality of Life in Adults with Osteogenesis Imperfecta. Calcif Tissue Int 101:473-478. https://doi.org/10.1007/s00223-0170301-4

8. Kruger KM, Caudill A, Rodriguez Celin M, et al (2019) Mobility in osteogenesis imperfecta: a multicenter North American study. Genet Med 0:1-8. https://doi.org/10.1038/s41436-0190491-4

9. Javaid MK, Forestier-Zhang L, Watts L, et al (2016) The RUDY study platform - a novel approach to patient driven research in rare musculoskeletal diseases. Orphanet J Rare Dis 11:19. https://doi.org/10.1186/s13023-016-0528-6

10. Ismail AA, O’Neill TW, Cockerill W, et al (2000) Validity of self-report of fractures: Results from a prospective study in men and women across Europe. Osteoporos Int 11:248-254. https://doi.org/10.1007/s001980050288

11. Ware JE, Sherbourne CD (1992) The MOS 36-item short-form health survey (Sf-36): I. conceptual framework and item selection. Med Care 30:473-483. https://doi.org/10.1097/00005650-199206000-00002

12. Jenkinson C, Coulter A, Wright L (1993) Short form 36 (SF36) health survey questionnaire: normative data for adults of working age. BMJ 306:1437-1440. https://doi.org/10.1136/bmj.306.6890.1437

13. Jenkinson C (1999) Comparison of UK and US methods for weighting and scoring the SF-36 
summary measures. J Public Health Med 21:372-376. https://doi.org/10.1093/pubmed/21.4.372

14. Rabin R, Charro F de (2001) EQ-SD: a measure of health status from the EuroQol Group. Ann Med 33:337-343. https://doi.org/10.3109/07853890109002087

15. Feng Y, Devlin N, Herdman M (2015) Assessing the health of the general population in England: how do the three- and five-level versions of EQ-5D compare? Health Qual Life Outcomes 13:171. https://doi.org/10.1186/s12955-015-0356-8

16. Melzack R (1975) The McGill Pain Questionnaire: major properties and scoring methods. Pain 1:277-99. https://doi.org/10.1016/0304-3959(75)90044-5

17. Yellen SB, Cella DF, Webster K, et al (1997) Measuring fatigue and other anemia-related symptoms with the Functional Assessment of Cancer Therapy (FACT) measurement system. J Pain Symptom Manage 13:63-74. https://doi.org/10.1016/S0885-3924(96)00274-6

18. Cella D, Lai JS, Chang CH, et al (2002) Fatigue in cancer patients compared with fatigue in the general United States population. Cancer 94:528-538. https://doi.org/10.1002/cncr.10245

19. Green J, Young J (2001) A test-retest reliability study of the Barthel Index, the Rivermead Mobility Index, the Nottingham Extended Activities of Daily Living Scale and the Frenchay Activities Index in stroke patients. Disabil Rehabil 23:670-676. https://doi.org/10.1080/09638280110045382

20. Engelbert RHH, Uiterwaal CSPM, Gulmans VAM, et al (2000) Osteogenesis imperfecta in childhood: Prognosis for walking. J Pediatr 137:397-402. https://doi.org/10.1067/mpd.2000.107892

21. Wekre LL, Frøslie KF, Haugen L, Falch JA (2010) A population-based study of demographical variables and ability to perform activities of daily living in adults with osteogenesis imperfecta. Disabil Rehabil 32:579-587. https://doi.org/10.3109/09638280903204690

22. Harsevoort AGJ, Gooijer K, van Dijk FS, et al (2020) Fatigue in adults with Osteogenesis Imperfecta. BMC Musculoskelet Disord 21:6. https://doi.org/10.1186/s12891-019-3000-7

23. Metsios GS, Stavropoulos-Kalinoglou A, van Zanten JJCSV, et al (2008) Rheumatoid arthritis, cardiovascular disease and physical exercise: A systematic review. Rheumatology 47:239248. https://doi.org/10.1093/rheumatology/kem260

24. Focht BC (2006) Effectiveness of exercise interventions in reducing pain symptoms among older adults with knee osteoarthritis: A review. J Aging Phys Act 14:212-235. https://doi.org/10.1123/japa.14.2.212

25. Lafage-Proust MH, Courtois I (2019) The management of osteogenesis imperfecta in adults: state of the art. Jt Bone Spine 1-5. https://doi.org/10.1016/j.jbspin.2019.02.001 


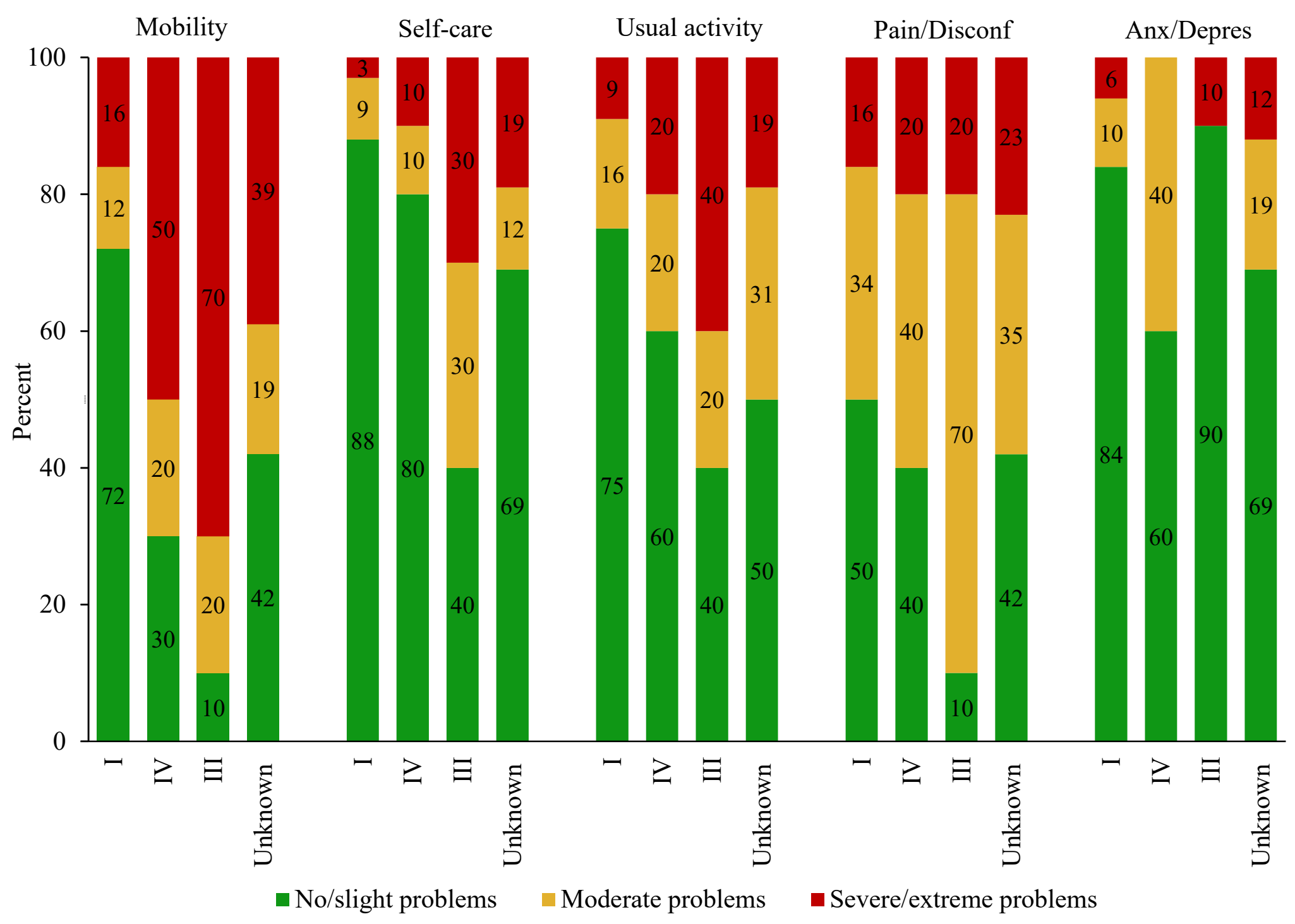


Table 1. Clinical features of study participants, as a whole and stratified by OI type

\begin{tabular}{|c|c|c|c|c|c|c|}
\hline & \multirow[b]{2}{*}{ All } & \multicolumn{4}{|c|}{ OI type } & \multirow[b]{2}{*}{$p$ values } \\
\hline & & I & IV & III & unknown & \\
\hline Sample size (n) & 78 & 32 & 10 & 10 & 26 & \\
\hline Age (years) & $43.5 \pm 14.5$ & $45.7 \pm 13.7$ & $40.7 \pm 18.8$ & $34.6 \pm 11.3$ & $45.3 \pm 14.2$ & 0.090 \\
\hline Age (range years) & $18-75$ & $21-71$ & $22-73$ & $24-65$ & $21-75$ & \\
\hline Gender, m/f (n) & $20 / 58$ & $8 / 24$ & $2 / 8$ & $3 / 7$ & $7 / 19$ & \\
\hline Fractures (n) & $15.4 \pm 16.6$ & $13.2 \pm 8.7$ & $11.1 \pm 12.4$ & $26 \pm 20.4$ & $16.5 \pm 23.5$ & 0.295 \\
\hline \multicolumn{7}{|l|}{ McGill pain scores } \\
\hline Non-neuropathic & $1.62(0.81 ; 3.08)$ & $1.70(0.52 ; 3.02)$ & $1.16(0.56 ; 2.22)$ & $1.54(0.54 ; 4.28)$ & $1.91(1.16 ; 2.88)$ & 0.512 \\
\hline Neuropathic & $1.20(0.27 ; 2.55)$ & $0.60(0.20 ; 2.70)$ & $0.95(0.15 ; 2.47)$ & $1.35(0.37 ; 2.35)$ & $1.80(0.60 ; 2.72)$ & 0.499 \\
\hline Affective & $1.80(0.45 ; 3.12)$ & $1.15(0.05 ; 3.25)$ & $1.15(0.22 ; 2.82)$ & $2.30(0.72 ; 3.20)$ & $2.15(1.22 ; 3.62)$ & 0.252 \\
\hline Total & $1.71(0.74 ; 2.86)$ & $1.75(0.43 ; 2.80)$ & $1.26(0.54 ; 1.91)$ & $1.54(1.12 ; 3.33)$ & $1.85(1.12 ; 3.19)$ & 0.340 \\
\hline FACIT-Fatigue & $33.5(20.7 ; 41)$ & $38.5(29 ; 44.7)$ & $26(17.2 ; 34.5)$ & $33.5(13.5 ; 37.5)$ & $25.5(19 ; 37.7)$ & 0.068 \\
\hline
\end{tabular}

Data expressed as mean \pm standard deviation or median and interquartile range as appropriate. 
Table 2 SF-36 physical component and domains scores in OI types and in the general population

\begin{tabular}{|c|c|c|c|}
\hline & type I & UK norm & \\
\hline & Mean (SD) & Mean (SD) & $p$ values \\
\hline \multicolumn{4}{|l|}{ Physical domains } \\
\hline Physical function & $57.3(28.4)$ & $87.2(20.9)$ & $<0.001$ \\
\hline Role physical & $41.4(45.1)$ & $83.5(33.8)$ & $<0.001$ \\
\hline Bodily Pain & $55.1(24.1)$ & $80.7(25.5)$ & $=0.001$ \\
\hline General Health & $47(14)$ & $71.3(23.4)$ & $=0.001$ \\
\hline \multirow[t]{2}{*}{ Physical component score } & $37(9.8)$ & $50(10)$ & $<0.001$ \\
\hline & type IV & UK norm & \\
\hline \multicolumn{4}{|l|}{ Physical domains } \\
\hline Physical function & $32(27.3)$ & $93.3(13.4)$ & $<0.001$ \\
\hline Role physical & $32.5(39.1)$ & $88.2(28)$ & $<0.001$ \\
\hline Bodily Pain & $51.9(23.6)$ & $84.9(21.8)$ & $<0.001$ \\
\hline General Health & $45.2(15.5)$ & $75.5(19.5)$ & $=0.001$ \\
\hline \multirow[t]{2}{*}{ Physical component score } & $29.8(12.9)$ & $50(10)$ & $<0.001$ \\
\hline & type III & UK norm & \\
\hline \multicolumn{4}{|l|}{ Physical domains } \\
\hline Physical function & $15.5(10.6)$ & $93.3(13.4)$ & $<0.001$ \\
\hline Role physical & $17.5(26.4)$ & $88.2(28)$ & $<0.001$ \\
\hline Bodily Pain & $40.9(20.3)$ & $84.9(21.8)$ & $<0.001$ \\
\hline General Health & $50.2(10)$ & $75.5(19.5)$ & $=0.001$ \\
\hline \multirow[t]{2}{*}{ Physical component score } & $25.5(4.9)$ & $50(10)$ & $<0.001$ \\
\hline & type unknown & UK norm & \\
\hline \multicolumn{4}{|l|}{ Physical domains } \\
\hline Physical function & $31.9(31.5)$ & $87.2(20.9)$ & $<0.001$ \\
\hline Role physical & $32.7(40.4)$ & $83.5(33.8)$ & $<0.001$ \\
\hline Bodily Pain & $50(23.3)$ & $80.7(25.5)$ & $=0.001$ \\
\hline General Health & $49.5(11.3)$ & $71.3(23.4)$ & $=0.001$ \\
\hline Physical component score & $31.6(11.1)$ & $50(10)$ & $<0.001$ \\
\hline
\end{tabular}


Table 3. Nottingham Extended Activities of Daily Living scores in people with OI.

\begin{tabular}{|l|c|c|c|c|c|c|c|c|c|}
\hline & \multicolumn{2}{|c|}{ type I } & \multicolumn{2}{c|}{ type IV } & \multicolumn{2}{c|}{ type III } & \multicolumn{2}{c|}{ type unknown } & $p$ values \\
\cline { 2 - 10 } & Median & IQR & Median & IQR & Median & IQR & Median & IQR & \\
\hline Mobility & 6 & $5-6$ & 3 & $1-6$ & 1 & $0-3$ & 5 & $1-6$ & $<0.001$ \\
\hline Domestic & 5 & $5-5$ & 3.5 & $2-5$ & 2.5 & $1-5$ & 3.5 & $2-5$ & 0.001 \\
\hline Kitchen & 5 & $5-5$ & 5 & $4-5$ & 5 & $2-5$ & 5 & $3-5$ & 0.316 \\
\hline Leisure & 5 & $5-6$ & 5 & $3-5$ & 4 & $3-5$ & 4 & $3-6$ & 0.018 \\
\hline Total & 21 & $19-22$ & 15.5 & $12-19$ & 11.5 & $7-18$ & 15 & $11-22$ & 0.001 \\
\hline
\end{tabular}


Table 4. Pearson/ Spearman correlation used for univariate analysis and multiple linear regression for multivariate analyses

\begin{tabular}{lccccc}
\hline \multicolumn{1}{c}{ PCS } & \multicolumn{2}{c}{ Univariate Analysis } & \multicolumn{3}{c}{ Multivariate analysis } \\
\hline Variables & Rho & $p$ values & beta & $95 \%$ CI & $p$ values \\
\hline Age & -0.114 & 0.320 & -0.226 & $-0.29,-0.04$ & 0.011 \\
Gender & -0.356 & 0.545 & - & - & - \\
Type of OI & -0.262 & 0.021 & -0.309 & $-5.12,-1.37$ & 0.001 \\
Number of fractures & -0.111 & 0.426 & - & - & - \\
Fatigue score & 0.519 & $<0.0001$ & 0.245 & $-4.34,-1.64$ & 0.021 \\
Non-neuropathic pain & -0.543 & $<0.0001$ & - & - & - \\
Neuropathic pain & -0.506 & $<0.0001$ & -0.439 & $0.03,0.37$ & $<0.0001$ \\
Affective pain & -0.460 & $<0.0001$ & - & - & - \\
Total pain score & -0.575 & $<0.0001$ & - & - & - \\
\hline
\end{tabular}

PCS: physical component score 